\title{
Hidden superconformal symmetry: Where does it come from?
}

\author{
Luis Inzunza* and Mikhail S. Plyushchay ${ }^{\dagger}$ \\ Departamento de Física, Universidad de Santiago de Chile, Casilla 307, Santiago 2, Chile
}

(Received 5 November 2017; published 5 February 2018)

\begin{abstract}
It is known that a single quantum harmonic oscillator is characterized by a hidden spectrum generating superconformal symmetry, but its origin has remained rather obscure. We show how this hidden superconformal symmetry can be derived by applying a nonlocal Foldy-Wouthuysen transformation to three extended systems with fermion degrees of freedom. The associated systems have essentially different nature from the point of view of conventional supersymmetric quantum mechanics, and generate the desired hidden symmetry in three different ways. We also trace out how the hidden superconformal symmetry of the quantum free particle system is produced in the limit of zero frequency.
\end{abstract}

DOI: 10.1103/PhysRevD.97.045002

\section{INTRODUCTION}

Supersymmetry relates bosons and fermions on the basis of $\mathbb{Z}_{2}$-graded superalgebras. Supersymmetry in quantum mechanics is implemented by separating symmetry generators into "even" ("bosonic") and "odd" ("fermionic") subsets. Coherently with this, the Hamiltonian's eigenstates are separated into "bosonic" and "fermionic" states. A standard implementation of supersymmetry in quantum mechanics is realized by introducing a set of fermion degrees of freedom in addition to the bosonic degrees of freedom described by usual coordinate and momentum operators. The fermionic set is realized by matrices of finite dimension, or in terms of Grassmann variables in superspace formulation of supersymmetric quantum mechanics $[1,2]$.

There exist, however, quantum mechanical systems in which supersymmetry is realized without introducing additional degrees of freedom. In such nonextended systems, space reflection can play a role of the $\mathbb{Z}_{2}$-grading operator [3-5]. The most known example of such a system corresponds, probably, to a one-dimensional quantum harmonic oscillator, in which there appears a spectrum generating hidden superconformal $\mathfrak{o} \mathfrak{g} \mathfrak{p}(1 \mid 2)$ symmetry [6-9]. This hidden superconformal symmetry has a further peculiarity in comparison with Witten's supersymmetric quantum mechanics: all its odd generators have a dynamical

\footnotetext{
*luis.inzunza@usach.cl

†mikhail.plyushchay@usach.cl
}

Published by the American Physical Society under the terms of the Creative Commons Attribution 4.0 International license. Further distribution of this work must maintain attribution to the author(s) and the published article's title, journal citation, and DOI. Funded by SCOAP ${ }^{3}$. character being explicitly depending on time integrals of motion of the system.

Thus, a hidden superconformal symmetry of the quantum harmonic oscillator has a rather obscure origin and nature from a perspective of conventional realization of supersymmetric quantum mechanics.

In the present paper we derive the hidden superconformal $\mathfrak{o} \mathfrak{g} \mathfrak{p}(1 \mid 2)$ symmetry of the quantum harmonic oscillator and its nonlocal $\mathfrak{o} \mathfrak{g} \mathfrak{p}(2 \mid 2)$ extension by applying a peculiar nonlocal Foldy-Wouthuysen transformation to three different associated systems with conventional quantum mechanical matrix fermion degrees of freedom. We also investigate a rather nontrivial transformation of the hidden superconformal symmetry of the harmonic oscillator into the corresponding superconformal symmetry of the quantum free particle system.

The paper is organized as follows. In Sec. II we describe the hidden superconformal $\mathfrak{o} \mathfrak{g} \mathfrak{p}(1 \mid 2)$ symmetry of the quantum harmonic oscillator and its nonlocal $\mathfrak{o} \mathfrak{s} \mathfrak{p}(2 \mid 2)$ extension. In Sec. III we consider an extended system with fermionic degrees of freedom that represents a doubled quantum harmonic oscillator. Such a system cannot be generated within a framework of a usual construction of supersymmetric quantum mechanics with supercharges anticommuting for the Hamiltonian. The extended system possesses the super-extended Schrödinger symmetry which under appropriate nonlocal Foldy-Wouthuysen transformation reduces and reproduces the hidden superconformal symmetry of the quantum harmonic oscillator. In Sec. IV we show how the hidden superconformal symmetry of the quantum oscillator can also be produced via the dual Darboux transformations. In Sec. $\mathrm{V}$ we consider the extended system obtained via a one-parametric two-step Darboux transformation of the quantum harmonic oscillator. Such a quantum system corresponds to the anomalyfree scheme of quantization of a classical system with the 
second-order supersymmetry and is described by a nonlinear super-extended Schrödinger symmetry. In the appropriate limit it reproduces the system from Sec. III in which time-independent supercharges anti-commute for the central charge of its Lie type super-extended Schrödinger symmetry. In Sec. VI we consider a rather peculiar zero frequency limit applied to the system from Sec. III and show how the hidden superconformal symmetry of the free quantum particle is generated. Section VII is devoted to the discussion and outlook.

\section{HIDDEN SUPERCONFORMAL SYMMETRY OF THE QUANTUM HARMONIC OSCILLATOR}

In a system of units with Plank constant $\hbar=1$, frequency $\omega=1$ and mass $m=\frac{1}{2}$, Hamiltonian of the quantum harmonic oscillator is

$$
L=-\frac{d^{2}}{d x^{2}}+x^{2}
$$

and its spectrum is given by a discrete set $E_{n}=2 n+1$, $n=0,1, \ldots$. We take ladder operators in a form

$$
a^{ \pm}=\mp \frac{d}{d x}+x, \quad\left[a^{+}, a^{-}\right]=2 .
$$

They anticommute with reflection operator $\mathcal{R}$ defined by $\mathcal{R}^{2}=1, \mathcal{R} x=-x \mathcal{R}$, and their anticommutator generates the Hamiltonian, $\left\{a^{+}, a^{-}\right\}=2 L$. Taking $\mathcal{R}$ as a $\mathbb{Z}_{2^{-}}$ grading operator, we identify the $a^{ \pm}$as odd, fermionic operators, $\left\{\mathcal{R}, a^{ \pm}\right\}=0$. Hamiltonian $L$ and quadratic operators $\left(a^{ \pm}\right)^{2}=\frac{d^{2}}{d x^{2}}+x^{2}+1 \mp 2 x \frac{d}{d x}$ are identified then as even, bosonic operators, $[\mathcal{R}, L]=0,\left[\mathcal{R},\left(a^{ \pm}\right)^{2}\right]=0$. This set of operators generates a Lie superalgebra in which commutators of even and odd generators with Hamiltonian are nontrivial: $\left[L, a^{ \pm}\right]= \pm 2 a^{ \pm},\left[L,\left(a^{ \pm}\right)^{2}\right]= \pm 4\left(a^{ \pm}\right)^{2}$. Consequently, operators $a^{ \pm}$and $\left(a^{ \pm}\right)^{2}$ are not integrals of motion in the sense of Heisenberg equations of motion $\frac{d}{d t} A=\frac{\partial}{\partial t} A-i[A, L]$. The operators "dressed" by a unitary evolution operator $U(t)=\exp (i L t)$ give the corresponding even and odd explicitly depending on time integrals of motion $U^{-1}(t) a^{ \pm} U(t)$ and $U^{-1}(t)\left(a^{ \pm}\right)^{2} U(t)$. We shall refer to integrals of such a nature as to dynamical integrals. Introducing the rescaled operators

$$
J_{0}=\frac{1}{4} L, \quad J_{ \pm}=\frac{1}{4} e^{\mp 4 i t}\left(a^{ \pm}\right)^{2}, \quad \alpha_{ \pm}=\frac{1}{4} e^{\mp i 2 t} a^{ \pm},
$$

we obtain the superalgebra with nontrivial (anti)commutation relations

$$
\left[J_{0}, J_{ \pm}\right]= \pm J_{ \pm}, \quad\left[J_{-}, J_{+}\right]=2 J_{0}
$$

$$
\begin{aligned}
& \left\{\alpha_{+}, \alpha_{-}\right\}=\frac{1}{2} J_{0}, \quad\left\{\alpha_{ \pm}, \alpha_{ \pm}\right\}=\frac{1}{2} J_{ \pm}, \\
& {\left[J_{0}, \alpha_{ \pm}\right]= \pm \frac{1}{2} \alpha_{ \pm}, \quad\left[J_{ \pm}, \alpha_{\mp}\right]=\mp \alpha_{ \pm} .}
\end{aligned}
$$

The superalgebra (2.4), (2.5), (2.6) describes the hidden superconformal $\mathfrak{o} \mathfrak{s p}(1 \mid 2)$ symmetry of the quantum harmonic oscillator [6,7]. The set of even integrals $J_{0}, J_{ \pm}$ generates the $\mathfrak{g l}(2, \mathbb{R})$ subalgebra (2.4), and relations (2.6) mean that fermionic generators $\alpha_{ \pm}$form a spin-1/2 representation of this Lie sub-algebra. All the generators of the $\mathfrak{o} \mathfrak{s} \mathfrak{p}(1 \mid 2)$ superconformal algebra are local in $x$ operators. The grading operator $\mathcal{R}$, being even timeindependent integral of motion, can be presented in a form $\mathcal{R}=\sin \left(\frac{\pi}{2} L\right)$ which explicitly reveals its non-local nature. Expanding the set of local integrals (2.3) by nonlocal timeindependent even integral $\mathcal{R}$ and by dynamical odd integrals

$$
\beta_{ \pm}=i \mathcal{R} \alpha_{ \pm}
$$

we extend the $\mathfrak{o} \mathfrak{s} \mathfrak{p}(1 \mid 2)$ for $\mathfrak{b} \mathfrak{g} \mathfrak{p}(2 \mid 2)$ superconformal algebra in which we have additionally the following nontrivial (anti)commutation relations which involve the nonlocal integrals $\mathcal{R}$ and $\beta_{ \pm}$:

$$
\begin{gathered}
{\left[J_{0}, \beta_{ \pm}\right]= \pm \frac{1}{2} \beta_{ \pm}, \quad\left[J_{ \pm}, \beta_{\mp}\right]=\mp \beta_{ \pm},} \\
\left\{\beta_{ \pm}, \beta_{ \pm}\right\}=\frac{1}{2} J_{ \pm}, \quad\left\{\beta_{+}, \beta_{-}\right\}=\frac{1}{2} J_{0}, \quad\left\{\alpha_{ \pm}, \beta_{\mp}\right\}=\mp \frac{i}{2} Z \\
{\left[Z, \alpha_{ \pm}\right]=\frac{i}{2} \beta_{ \pm}, \quad\left[Z, \beta_{ \pm}\right]=-\frac{i}{2} \alpha_{ \pm},}
\end{gathered}
$$

where we introduced a notation

$$
Z=-\frac{1}{4} \mathcal{R}
$$

In terms of linear combinations

$$
\gamma_{ \pm}=\alpha_{ \pm}+i \beta_{ \pm}, \quad \delta_{ \pm}=\gamma_{\mp}^{\dagger}=\alpha_{ \pm}-i \beta_{ \pm},
$$

a part of superalgebra involving odd generators can be presented in an alternative form

$$
\begin{array}{rlrl}
{\left[J_{0}, \gamma_{ \pm}\right]} & = \pm \frac{1}{2} \gamma_{ \pm}, & {\left[J_{0}, \delta_{ \pm}\right]} & = \pm \frac{1}{2} \delta_{ \pm}, \\
{\left[J_{ \pm}, \gamma_{\mp}\right]} & =\mp \gamma_{ \pm}, \quad\left[J_{ \pm}, \delta_{\mp}\right]=\mp \delta_{ \pm},
\end{array}
$$




$$
\begin{aligned}
{\left[Z, \gamma_{ \pm}\right] } & =\frac{1}{2} \gamma_{ \pm}, \quad\left[Z, \delta_{ \pm}\right]=-\frac{1}{2} \delta_{ \pm}, \\
\left\{\gamma_{ \pm}, \delta_{ \pm}\right\} & =J_{ \pm}, \quad\left\{\gamma_{ \pm}, \delta_{\mp}\right\}=J_{0} \mp Z .
\end{aligned}
$$

\section{EXTENDED SYSTEM WITH SUPER- SCHRÖDINGER SYMMETRY AND NONLOCAL FOLDY-WOUTHUYSEN TRANSFORMATION}

Now let us show how the described hidden superconformal $\mathfrak{o} \mathfrak{a p}(1 \mid 2)$ and $\mathfrak{o} \mathfrak{g} \mathfrak{p}(2 \mid 2)$ symmetries of a single oscillator can be 'extracted' from the super-Schrödinger symmetry of the extended quantum harmonic oscillator system described by the Hamiltonian

$$
\mathcal{H}=\left(\begin{array}{ll}
L & 0 \\
0 & L
\end{array}\right)
$$

This system represents two copies of the quantum harmonic oscillator (2.1), and has three obvious matrix integrals of motion given by the Pauli matrices $\sigma_{1}, \sigma_{2}$ and $\sigma_{3}$. It is natural to identify the diagonal matrix $\Gamma=\sigma_{3}$ as a $\mathbb{Z}_{2}$-grading operator. Then Hamiltonian (3.1) is identified as even generator and the antidiagonal integrals $\sigma_{a}, a=1,2$, can be considered as odd supercharges. The peculiarity of the system (3.1) is that the supercharges $\sigma_{a}$ anticommute not for Hamiltonian but for central element, $\left\{\sigma_{a}, \sigma_{b}\right\}=2 \delta_{a b} \square, \square=\operatorname{diag}(1,1)$. All the energy levels of the extended system (2.1) including the lowest nonzero energy level $E_{0}=1>0$ are doubly degenerate, and the Witten index of this extended system equals zero. The doublet of states corresponding to the lowest energy level is not annihilated by supercharges $\sigma_{a}$, and (2.1) is identified as a quantum mechanical supersymmetric system in a phase of spontaneously broken supersymmetry. The time-independent even, $\mathcal{H}$ and $\sigma_{3}$, and odd, $\sigma_{1}$ and $\sigma_{2}$, integrals of motion together with even central charge 『 are local operators. Besides them, the system (3.1) also has local dynamical integrals of motion

$$
\begin{aligned}
& \mathcal{J}_{ \pm}= \frac{1}{4} e^{\mp i 4 t}\left(\begin{array}{cc}
\left(a^{ \pm}\right)^{2} & 0 \\
0 & \left(a^{ \pm}\right)^{2}
\end{array}\right)=\left(\begin{array}{cc}
J_{ \pm} & 0 \\
0 & J_{ \pm}
\end{array}\right), \\
& \mathcal{C}_{ \pm}=\frac{1}{4} e^{\mp i 2 t}\left(\begin{array}{cc}
a^{ \pm} & 0 \\
0 & a^{ \pm}
\end{array}\right)=\left(\begin{array}{cc}
\alpha_{ \pm} & 0 \\
0 & \alpha_{ \pm}
\end{array}\right), \\
& \mathcal{Q}_{ \pm}=\frac{1}{4} e^{\mp i 2 t}\left(\begin{array}{cc}
0 & a^{ \pm} \\
a^{ \pm} & 0
\end{array}\right)=\left(\begin{array}{cc}
0 & \alpha_{ \pm} \\
\alpha_{ \pm} & 0
\end{array}\right), \\
& \mathcal{S}_{ \pm}=i \sigma_{3} \mathcal{Q}_{ \pm} .
\end{aligned}
$$

Diagonal operators $\mathcal{J}_{ \pm}$and $\mathcal{C}_{ \pm}$are identified here as even generators, and antidiagonal dynamical integrals $\mathcal{Q}_{ \pm}$and $\mathcal{S}_{ \pm}$are odd generators. All these integrals generate the superalgebra with the following (anti)commutation relations:

$$
\begin{array}{r}
{\left[\mathcal{J}_{0}, \mathcal{J}_{ \pm}\right]= \pm \mathcal{J}_{ \pm}, \quad\left[\mathcal{J}_{-}, \mathcal{J}_{+}\right]=2 \mathcal{J}_{0},} \\
{\left[\mathcal{J}_{0}, \mathcal{C}_{ \pm}\right]= \pm \frac{1}{2} \mathcal{C}_{ \pm}, \quad\left[\mathcal{J}_{ \pm}, \mathcal{C}_{\mp}\right]=\mp \mathcal{C}_{ \pm}, \quad\left[\mathcal{C}_{-}, \mathcal{C}_{+}\right]=\frac{1}{2} \mathcal{I}}
\end{array}
$$

$$
\begin{array}{ll}
{\left[\mathcal{J}_{0}, \mathcal{Q}_{ \pm}\right]= \pm \frac{1}{2} \mathcal{Q}_{ \pm},} & {\left[\mathcal{J}_{0}, \mathcal{S}_{ \pm}\right]= \pm \frac{1}{2} \mathcal{S}_{ \pm},} \\
{\left[\mathcal{J}_{ \pm}, \mathcal{Q}_{\mp}\right]=\mp \mathcal{Q}_{ \pm}, \quad\left[\mathcal{J}_{ \pm}, \mathcal{S}_{\mp}\right]=\mp \mathcal{S}_{ \pm}} \\
\left\{\Sigma_{a}, \Sigma_{b}\right\}=2 \delta_{a b} \mathcal{I}, & \left\{\Sigma_{1}, \mathcal{Q}_{ \pm}\right\}=\mathcal{C}_{ \pm} \\
\left\{\Sigma_{2}, \mathcal{S}_{ \pm}\right\} & =\mathcal{C}_{ \pm},
\end{array}
$$$$
\left\{\mathcal{Q}_{ \pm}, \mathcal{Q}_{ \pm}\right\}=\frac{1}{2} \mathcal{J}_{ \pm}, \quad\left\{\mathcal{Q}_{+}, \mathcal{Q}_{-}\right\}=\frac{1}{2} \mathcal{J}_{0}
$$$$
\left\{\mathcal{S}_{ \pm}, \mathcal{S}_{ \pm}\right\}=\frac{1}{2} \mathcal{J}_{ \pm}, \quad\left\{\mathcal{S}_{+}, \mathcal{S}_{-}\right\}=\frac{1}{2} \mathcal{J}_{0},
$$$$
\left\{\mathcal{Q}_{+}, \mathcal{S}_{-}\right\}=-\frac{i}{2} \mathcal{Z}, \quad\left\{\mathcal{Q}_{-}, \mathcal{S}_{+}\right\}=\frac{i}{2} \mathcal{Z}
$$

$\left[\mathcal{Z}, \Sigma_{a}\right]=\frac{i}{2} \epsilon_{a b} \Sigma_{b}, \quad\left[\mathcal{Z}, \mathcal{Q}_{ \pm}\right]=\frac{i}{2} \mathcal{S}_{ \pm}, \quad\left[\mathcal{Z}, \mathcal{S}_{ \pm}\right]=-\frac{i}{2} \mathcal{Q}_{ \pm}$,

$$
\left[\mathcal{C}_{ \pm}, \mathcal{Q}_{\mp}\right]=\mp \frac{1}{4} \Sigma_{1}, \quad\left[\mathcal{C}_{ \pm}, \mathcal{S}_{\mp}\right]=\mp \frac{1}{4} \Sigma_{2},
$$

where $\mathcal{J}_{0}$ is a rescaled Hamiltonian (3.1),

$$
\mathcal{J}_{0}=\frac{1}{4} \mathcal{H}=\left(\begin{array}{cc}
J_{0} & 0 \\
0 & J_{0}
\end{array}\right)
$$

and we introduced the notation

$\Sigma_{1}=\frac{1}{2} \sigma_{1}, \quad \Sigma_{2}=-\frac{1}{2} \sigma_{2}, \quad \mathcal{Z}=-\frac{1}{4} \sigma_{3}, \quad \mathcal{I}=\frac{1}{4} \rrbracket$.

The not shown (anti)commutators between generators are equal to zero. By comparing this superalgebra and the structure of its generators with superalgebra and generators of the hidden superconformal $\mathfrak{o} \mathfrak{s} \mathfrak{p}(1 \mid 2)$ and $\mathfrak{o} \mathfrak{s} \mathfrak{p}(2 \mid 2)$ symmetries of the quantum harmonic oscillator, it is obvious that the matrix integrals $\mathcal{J}_{0}, \mathcal{J}_{ \pm}, \mathcal{Z}, \mathcal{Q}_{ \pm}, \mathcal{S}_{ \pm}$of the extended system (3.1) are analogous to the corresponding integrals $J_{0}$, $J_{ \pm}, Z, \alpha_{ \pm}, \beta_{ \pm}$of the quantum harmonic oscillator. Because of the extension, the nonlocal integrals $Z$ and $\beta_{ \pm}$of the system (2.1) are changed here for the corresponding local matrix integrals $\mathcal{Z}$ and $\mathcal{S}_{ \pm}$. The anticommutator of additional fermionic integrals $\Sigma_{a}$ with $\Sigma_{b}$ generates a central charge $\mathcal{I}$, and via the anticommutators with odd dynamical integrals $\mathcal{Q}_{ \pm}$and $\mathcal{S}_{ \pm}$they produce additional bosonic integrals $\mathcal{C}_{ \pm}$, see Eq. (3.8). The superalgebra (3.5)-(3.12) 
represents a super-extended Schrödinger symmetry of the matrix system (3.1) with relations (3.5), (3.7), (3.9) and (3.10) corresponding to $\mathfrak{o} \mathfrak{g} \mathfrak{p}(2 \mid 2)$ sub-superalgebra.

The comparison of the symmetries and generators of the systems (3.1) and (2.1) indicates that the local $\mathfrak{o} \mathfrak{g} \mathfrak{p}(1 \mid 2)$ and nonlocal $\mathfrak{o} \mathfrak{s} \mathfrak{p}(2 \mid 2)$ hidden superconformal symmetries of the quantum harmonic oscillator can be obtained by a certain projection (reduction) of the local symmetries of the matrix system (3.1). To find the exact relation between these two systems and their symmetries, we apply to the extended system a unitary transformation $\mathcal{O} \mapsto \tilde{\mathcal{O}}=$ $U \mathcal{O} U^{\dagger}$ generated by the nonlocal matrix operator

$$
U=U^{\dagger}=U^{-1}=\frac{1}{2}\left(\begin{array}{ll}
1+\mathcal{R} & 1-\mathcal{R} \\
1-\mathcal{R} & 1+\mathcal{R}
\end{array}\right) .
$$

This transformation acts in the following way on the basic operators of the matrix system (3.1): $\tilde{x}=x \sigma_{1}, \tilde{p}=p \sigma_{1}$, $\widetilde{\sigma_{1}}=\sigma_{1}, \widetilde{\sigma_{2}}=\sigma_{2} \mathcal{R}$ and $\widetilde{\sigma_{3}}=\sigma_{3} \mathcal{R}$, and we also have $\tilde{\mathcal{R}}=\mathcal{R}$. As a consequence, the central element $\mathcal{I}$ and generators of the $\mathfrak{I l}(2, \mathbb{R})$ subalgebra, $\mathcal{J}_{0}$ and $\mathcal{J}_{ \pm}$, do not change under this transformation, while other generators take the following form:

$$
\begin{gathered}
\tilde{Z}=\frac{1}{4}\left(\begin{array}{cc}
-\mathcal{R} & 0 \\
0 & \mathcal{R}
\end{array}\right), \\
\widetilde{\mathcal{Q}_{ \pm}}=\left(\begin{array}{cc}
\alpha_{ \pm} & 0 \\
0 & \alpha_{ \pm}
\end{array}\right), \\
\widetilde{\mathcal{S}_{ \pm}}=\left(\begin{array}{cc}
i \mathcal{R} \alpha_{ \pm} & 0 \\
0 & -i \mathcal{R} \alpha_{ \pm}
\end{array}\right)=\left(\begin{array}{cc}
\beta_{ \pm} & 0 \\
0 & -\beta_{ \pm}
\end{array}\right), \\
\widetilde{\Sigma_{1}}=\frac{1}{2} \sigma_{1}, \quad \widetilde{\Sigma_{2}}=-\frac{1}{2} \sigma_{2} \mathcal{R}, \quad \widetilde{\mathcal{C}_{ \pm}}=\sigma_{1} \alpha_{ \pm} .
\end{gathered}
$$

The unitary transformation generated by nonlocal operator (3.15) diagonalizes the dynamical odd integrals $\mathcal{Q}_{ \pm}$and $\mathcal{S}_{ \pm}$ which initially have had the antidiagonal form. The transformation therefore is of a nature of Foldy-Wouthuysen transformation for Dirac particle in external electric and magnetic fields [10]. It is interestingly to note that the transformed odd and even integrals $\widetilde{\mathcal{Q}_{ \pm}}$and $\widetilde{\mathcal{C}_{ \pm}}$take the form of the original even and odd integrals $\mathcal{C}_{ \pm}$and $\mathcal{Q}_{ \pm}$, respectively. The transformed even, $\tilde{\mathcal{Z}}$, and odd, $\widetilde{\mathcal{S}_{ \pm}}$, generators of the superconformal $\mathfrak{o} \mathfrak{s} \mathfrak{p}(2,2)$ sub-superalgebra of the super-extended Schrödinger symmetry of the system (3.1) take a nonlocal form. We can reduce (or, in other words, project) the transformed system and its symmetries to the proper subspace of eigenvalue +1 of the matrix $\sigma_{3}=\widetilde{\mathcal{R} \sigma_{3}}$ which corresponds, according to Eq. (3.13), to the single (non-extended) quantum harmonic oscillator system. This can be done by multiplying all the transformed generators $\widetilde{\mathcal{X}}_{i}$ of the super-extended
Schrödinger symmetry from both sides by the projector $\Pi_{+}=\frac{1}{2}\left(1+\sigma_{3}\right): \widetilde{\mathcal{X}}_{i} \mapsto \Pi_{+} \widetilde{\mathcal{X}}_{i} \Pi_{+}$. Since the transformed generators $\widetilde{\Sigma_{a}}$ and $\widetilde{\mathcal{C}_{ \pm}}$are anti-diagonal and anticommute with $\sigma_{3}$, we loose them in the reduction procedure by mapping them into zero. Since the central element $\mathcal{I}$ is generated in superalgebra of the super-extended Schrödinger symmetry of the system (3.1) via the anticommutators of $\widetilde{\Sigma_{a}}$ with $\widetilde{\Sigma_{b}}$ and commutator of $\widetilde{\mathcal{C}_{+}}$and $\widetilde{\mathcal{C}_{-}}$, we also loose it as a generator of the surviving part of the superalgebra. The rest of the generators in the proper subspace of eigenvalue +1 of $\sigma_{3}$ will take exactly the form of the corresponding generators of the hidden superconformal $\mathfrak{o} \mathfrak{s} \mathfrak{p}(2,2)$ symmetry of the quantum harmonic oscillator system (2.1).

\section{SUPERCONFORMAL SYMMETRY VIA DUAL DARBOUX TRANSFORMATIONS}

Though the extended system (3.1) has allowed us to derive the hidden superconformal symmetry of the quantum harmonic oscillator, it cannot be generated directly within the framework of supersymmetric quantum mechanics with its underlying structure of Darboux transformations. Nevertheless, we shall show in this section how the super-extended Schrödinger symmetry can be generated via a usual supersymmetric extension of the quantum harmonic oscillator, and trace out a difference in the associated reduction procedure leading to the hidden superconformal symmetry. ${ }^{1}$

The harmonic oscillator corresponds to the simplest case of the duality induced by Darboux transformations [13]. This means that the same, modulo an additive shift, superpartner can be produced for a given system by choosing different states as seed states to generate a Darboux transformation. The presence of a nontrivial relative shift will play, as we shall see, a crucial role in generating the structure of superextended Schrödinger symmetry.

Let us construct the Darboux intertwining operators for the quantum harmonic oscillator by taking its ground state $\psi_{0}=C e^{-x^{2} / 2}$ as a seed state, where a concrete value of a normalization constant $C$ is of no importance. They are nothing else as the ladder operators,

$$
A_{0}^{-}=\psi_{0} \frac{d}{d x} \frac{1}{\psi_{0}}=a^{-}, \quad A_{0}^{+}=\left(A_{0}^{-}\right)^{\dagger}=a^{+},
$$

which generate two mutually shifted copies of the quantum harmonic oscillator: $A_{0}^{+} A_{0}^{-}=a^{+} a^{-}=L-1=H_{-}$and $A_{0}^{-} A_{0}^{+}=a^{-} a^{+}=L+1=H_{+}$. The superpartner system for $H_{-}$is therefore the same but shifted harmonic oscillator

\footnotetext{
${ }^{1}$ See also Refs. [11,12] for the discussion of superconformal symmetry of the superextended quantum harmonic oscillator obtained within the framework of conventional supersymmetric quantum mechanics construction.
} 
$H_{+}=H_{-}+2$. The operators $A_{0}^{ \pm}=a^{ \pm}$intertwine the super-partner systems $H_{-}$and $H_{+}$,

$$
A_{0}^{-} H_{-}=H_{+} A_{0}^{-}, \quad A_{0}^{+} H_{+}=H_{-} A_{0}^{+} .
$$

Together $H_{-}$and $H_{+}$constitute the extended Hamiltonian which can be presented in terms of a superpotential $W=-\left(\ln \psi_{0}\right)^{\prime}=x$,

$$
\hat{\mathcal{H}}=-\frac{d^{2}}{d x^{2}}+W^{2}+\sigma_{3} W^{\prime}=\left(\begin{array}{cc}
H_{+} & 0 \\
0 & H_{-}
\end{array}\right) .
$$

The intertwining operators constitute the building blocks for time-independent supercharges for the system (4.3),

$$
\hat{\mathcal{Q}}_{1}=\left(\begin{array}{cc}
0 & a^{-} \\
a^{+} & 0
\end{array}\right), \quad \hat{\mathcal{Q}}_{2}=i \sigma_{3} \hat{\mathcal{Q}}_{1},
$$

$\left[\hat{\mathcal{H}}, \hat{\mathcal{Q}}_{a}\right]=0, \quad\left\{\hat{\mathcal{Q}}_{a}, \hat{\mathcal{Q}}_{b}\right\}=2 \delta_{a b} \hat{\mathcal{H}}$, with integral $\Gamma=\sigma_{3}$ identified as a $\mathbb{Z}_{2}$-grading operator. Since a singlet ground state $\Psi_{0}=\left(0, \psi_{0}\right)^{T}$ of $\hat{\mathcal{H}}$ is annihilated by both supercharges $\hat{\mathcal{Q}}_{a}$, the system (4.3), unlike (3.1), corresponds to the case of exact, unbroken supersymmetry.

Instead of the ground state $\psi_{0}$, we can take a nonnormalizable (nonphysical) eigenstate $\psi_{0}^{-}=1 / \psi_{0}=$ $C^{-1} e^{x^{2} / 2}$ of eigenvalue $E_{-0}=-1$ of the quantum harmonic oscillator $L$ to generate the Darboux transformation. The corresponding operators in this case are

$$
A_{-0}^{-}=\psi_{0}^{-} \frac{d}{d x} \frac{1}{\psi_{0}^{-}}=-a^{+}, \quad A_{-0}^{+}=\left(A_{-0}^{-}\right)^{\dagger}=-a^{-} .
$$

They satisfy relations $A_{-0}^{+} A_{-0}^{-}=L+1=H_{+}, A_{-0}^{-} A_{-0}^{+}=$ $L-1=H_{-}, \quad A_{-0}^{-} H_{+}=H_{-} A_{-0}^{-}, \quad A_{-0}^{+} H_{-}=H_{+} A_{-0}^{+}$, and generate a supersymmetric system described by the Hamiltonian operator

$$
\breve{\mathcal{H}}=-\frac{d^{2}}{d x^{2}}+W^{2}-\sigma_{3} W^{\prime}=\left(\begin{array}{cc}
H_{-} & 0 \\
0 & H_{+}
\end{array}\right)
$$

which has two conserved supercharges

$$
\breve{\mathcal{S}}_{1}=\left(\begin{array}{cc}
0 & a^{+} \\
a^{-} & 0
\end{array}\right), \quad \breve{\mathcal{S}}_{2}=i \sigma_{3} \breve{\mathcal{S}}_{1},
$$

$\left[\breve{\mathcal{H}}, \breve{\mathcal{S}}_{a}\right]=0,\left\{\breve{\mathcal{S}}_{a}, \breve{\mathcal{S}}_{b}\right\}=2 \delta_{a b} \breve{\mathcal{H}}$. Hamiltonian (4.6) and its supercharges (4.7) are related to Hamiltonian (4.3) and its supercharges (4.4) by a unitary transformation generated by $\sigma_{1}$ :

$$
\breve{\mathcal{H}}=\sigma_{1} \hat{\mathcal{H}} \sigma_{1}, \quad \breve{\mathcal{S}}_{1}=\sigma_{1} \hat{\mathcal{Q}}_{1} \sigma_{1}, \quad-\breve{\mathcal{S}}_{2}=\sigma_{1} \hat{\mathcal{Q}}_{2} \sigma_{1} .
$$

The commutator of $\hat{\mathcal{H}}$ with $\sigma_{1}$ is $\left[\hat{\mathcal{H}}, \sigma_{1}\right]=2 \sigma_{3} \sigma_{1}$. Denoting by $\tau$ the parameter associated with evolution generated by
$\hat{\mathcal{H}}$, we find the dynamical integrals corresponding to $\sigma_{1}$ and $\quad \sigma_{2}=i \sigma_{3} \sigma_{1}: \quad \hat{\Sigma}_{a}(\tau)=\exp (-i \hat{\mathcal{H}} \tau) \hat{\Sigma}_{a}(0) \exp (i \hat{\mathcal{H}} \tau)=$ $e^{-i 2 \sigma_{3} \tau} \hat{\Sigma}_{a}(0), a=1,2$, where $\hat{\Sigma}_{a}(0)=\Sigma_{a}$, and $\Sigma_{a}$ are defined in (3.14). From here we also find that $\hat{\mathcal{S}}_{a}(\tau)=$ $e^{-4 i \sigma_{3} \tau} \breve{\mathcal{S}}_{a}$ are dynamical integrals for $\hat{\mathcal{H}}$.

Proceeding from the supercharges $\hat{\mathcal{Q}}_{a}$ and dynamical odd integrals $\hat{\Sigma}_{a}$ and $\hat{\mathcal{S}}_{a}$, we find the symmetry of the system $\hat{\mathcal{H}}$. Besides the Hamiltonian $\hat{\mathcal{H}}$ and the listed fermionic integrals, its set of generators also includes bosonic time-independent integrals $\mathcal{Z}=-\frac{1}{4} \sigma_{3}$ and $\mathcal{I}=\frac{1}{4} \llbracket$, and the even dynamical integrals of motion $\hat{\mathcal{J}}_{ \pm}(\tau)$ and $\hat{\mathcal{C}}_{ \pm}(\tau)$, which have the same form as $\mathcal{J}_{ \pm}$and $\mathcal{C}_{ \pm}$in (3.2) and (3.3) but with evolution parameter $t$ changed here for $\tau$. The Hamiltonian of the extended system (3.1) is related to supersymmetric Hamiltonian operators (4.3) and (4.6) by an equality

$$
\mathcal{H}=\frac{1}{2}(\hat{\mathcal{H}}+\breve{\mathcal{H}})=\hat{\mathcal{H}}-\sigma_{3} .
$$

In correspondence with this relation, we introduce a notation for a linear combination of Hamiltonian $\hat{\mathcal{H}}$ and bosonic integral $\mathcal{Z}$,

$$
\mathcal{J}_{0}=\frac{1}{4} \hat{\mathcal{H}}+\mathcal{Z} .
$$

As a result we find that the system given by the Hamiltonian operator (4.3) is described by the same super-extended Schrödinger symmetry (3.5)-(3.12) as the system (3.1) with the already identified relation between the even generators, while the correspondence between odd generators of both systems is given by $\Sigma_{a}=\hat{\Sigma}_{a}(0)$, and

$$
\begin{aligned}
& \mathcal{Q}_{ \pm}(0)=\left.\frac{1}{8}\left[\hat{\mathcal{Q}}_{1}+\hat{\mathcal{S}}_{1} \pm i\left(\hat{\mathcal{Q}}_{2}-\hat{\mathcal{S}}_{2}\right)\right]\right|_{\tau=0}, \\
& \mathcal{S}_{ \pm}(0)=\left.\frac{1}{8}\left[\hat{\mathcal{Q}}_{2}+\hat{\mathcal{S}}_{2} \mp i\left(\hat{\mathcal{Q}}_{1}-\hat{\mathcal{S}}_{1}\right)\right]\right|_{\tau=0} .
\end{aligned}
$$

Note that both systems (4.3) and (3.1) are characterized by the same number of time-independent and dynamical integrals. The key difference is that coherently with different structure of Hamiltonian operators related by Eq. (4.9), the system (3.1) has two time-independent integrals $\Sigma_{a}$ anticommuting for the central charge $\mathcal{I}$, while timeindependent integrals $\hat{\mathcal{Q}}_{a}$ of the system (4.3) anticommute for the Hamiltonian operator $\hat{\mathcal{H}}$. Another essential difference is that the even dynamical integrals $\mathcal{J}_{+}$and $\mathcal{J}_{-}$of the system (3.1) commute for the third generator $\mathcal{J}_{0}$ of $\mathfrak{g l}(2, \mathbb{R})$ subalgebra being the rescaled Hamiltonian $\mathcal{H}$, while the corresponding dynamical integrals $\hat{\mathcal{J}}_{+}$and $\hat{\mathcal{J}}_{-}$of the system (4.9) anticommute for a linear combination of its Hamiltonian $\hat{\mathcal{H}}$ and bosonic integral $\mathcal{Z}$. 
We can apply to the symmetry generators of the system described by the Hamiltonian $\hat{\mathcal{H}}$ the same unitary transformation generated by nonlocal operator (3.15) and then realize the reduction of the transformed operators by means of projection $\widetilde{\mathcal{X}_{i}} \mapsto \Pi_{+} \widetilde{\mathcal{X}}_{i} \Pi_{+}$to the proper eigenspace of eigenvalue +1 of $\sigma_{3}=\widetilde{\sigma_{3} \mathcal{R}}$. In this way we, again, reproduce the hidden superconformal symmetry of the single quantum harmonic oscillator system. The peculiarity in this case is, however, that according to relation (4.9), the application of unitary transformation and projection to the Hamiltonian $\hat{\mathcal{H}}$ results not in Hamiltonian of the harmonic oscillator operator $L$ but in a nonlocal operator: $\hat{\mathcal{H}} \mapsto L-\mathcal{R}$.

In conclusion of this section it is worth noting that like the Darboux generators (4.1) obtained from the ground state $\psi_{0}$, the operators (4.5) constructed on the base of nonphysical eigenstate $\psi_{-0}=1 / \psi_{0}$ intertwine $H_{-}$and $H_{+}$but with an additional shift:

$$
A_{-0}^{+} H_{-}=\left(H_{+}-4\right) A_{-0}^{+}, \quad A_{-0}^{-}\left(H_{+}-4\right)=H_{-} A_{-0}^{-} .
$$

It is because of such an additional shift matrix operators $\breve{\mathcal{S}}_{a}$, unlike $\hat{\mathcal{Q}}_{a}$, correspond to dynamical integrals of motion of the system $\hat{\mathcal{H}}$. In the case of reflectionless and finite-gap systems, there exist two pairs of operators that intertwine the corresponding partner systems exactly in the same way, without additional shift. As a consequence, instead of two time-independent supercharges, the Darboux-extended systems are characterized there by four time-independent supercharges, which generate the corresponding nontrivial Lax-Novikov integrals. For details see Ref. [14].

\section{TWO-STEP ISOSPECTRAL DARBOUX CHAIN}

The extended system (3.1) cannot be produced by a usual quantum mechanical supersymmetric construction of the form (4.3) based on some superpotential $W(x)$ that is equivalent to application of a one-step Darboux transformation. Let us show that it can be generated via a twostep isospectral Darboux chain, that uses a certain Jordan state of the quantum harmonic oscillator, with subsequent application of a simple limit procedure. The corresponding extended system obtained via the two-step isospectral Darboux chain possesses a set of time-independent and dynamical integrals of motion. After application of the limit procedure these integrals give us the generators of the superextended Schrödinger symmetry of the system (3.1).

Consider a Darboux-Crum transformation based on the seed states $\psi_{0}(x)$ and $\chi_{0}(x)$, where $\psi_{0}(x)$ is a normalized ground state of the quantum harmonic oscillator (2.1) and $\chi_{0}(x ; \mu)$ is its Jordan state of order two corresponding to the same energy $E=1$ [8],

$$
\chi_{0}(x ; \mu)=\widetilde{\mu \psi_{0}(x)}+\psi_{0}(x) \int_{0}^{x} \frac{1}{\left(\psi_{0}(t)\right)^{2}} I_{0}(t) d t
$$

Here $\mu$ is a real constant,

$$
\widetilde{\psi_{0}(x)}=\psi_{0}(x) \int_{0}^{x}\left(\psi_{0}(t)\right)^{-2} d t
$$

is a linear independent from $\psi_{0}(x)$ nonphysical eigenstate of $L$ of the same energy $E=1$, and

$$
I_{0}(x)=\int_{-\infty}^{x}\left(\psi_{0}(t)\right)^{2} d t
$$

is a monotonic function that varies between 0 and 1 as soon as the ground state wave function $\psi_{0}(x)$ is normalized for one. Note that the potential of the system shifted for corresponding eigenvalue $E=1$ is "extracted" from $I_{0}(x)$ by the Schwarzian: $-\frac{1}{2} S\left(I_{0}\right)=x^{2}-1$, where $S(f)=$ $\left(f^{\prime \prime \prime} / f^{\prime}\right)-\frac{3}{2}\left(f^{\prime \prime} / f^{\prime}\right)^{2}$. The application of the operator $a^{-}$ to the state $\chi_{0}(x ; \mu)$ produces the function

$$
a^{-} \chi_{0}(x ; \mu)=\frac{\mu+I_{0}(x)}{\psi_{0}(x)}=\varphi_{-0}(x ; \mu),
$$

that satisfies a relation $a^{+} \varphi_{-0}(x ; \mu)=-\psi_{0}(x)$. The state $\varphi_{-0}(x ; \mu)$ is a linear combination of the nonphysical eigenstate $\psi_{-0}(x)=1 / \psi_{0}(x)$ of $L$ of eigenvalue -1 , that we used before to generate the system (4.6), and of a linear independent nonphysical eigenstate $\widetilde{\psi_{-0}(x)}$ of $L$ of the same eigenvalue -1 constructed according to relation analogous to (5.2):

$$
\varphi_{-0}(x ; \mu)=\mu \psi_{-0}(x)+\widetilde{\psi_{-0}(x)} .
$$

We choose the value of parameter $\mu$ in one of the infinite intervals $(-\infty,-1)$ or $(0, \infty)$ for which $\varphi_{-0}(x ; \mu)$ is a nodeless on a real line function being a nonphysical eigenstate of $H_{+}=a^{-} a^{+}$of zero eigenvalue, $H_{+} \varphi_{-0}(x ; \mu)=0$. For the wave function $\chi_{0}(x ; \mu)$ we find equivalently that it satisfies a relation $a^{-} a^{+} a^{-} \chi_{0}(x ; \mu)=0$, and therefore $(L-1)^{2} \chi_{0}=0$. This means that $\chi_{0}(x ; \mu)$ is indeed the Jordan state of order two of $L=H_{-}+1$ corresponding to eigenvalue $E=1$.

We can use the eigenstate $\varphi_{-0}(x ; \mu)$ of $H_{+}$as a seed state for a new Darboux transformation which produces the first order differential operators

$$
A_{\mu}^{-}=\varphi_{-0}(x ; \mu) \frac{d}{d x} \frac{1}{\varphi_{-0}(x ; \mu)}=\frac{d}{d x}+W(x ; \mu), \quad A_{\mu}^{+}=\left(A_{\mu}^{-}\right)^{\dagger},
$$

where 


$$
W(x ; \mu)=-\left(\ln \varphi_{-0}(x ; \mu)\right)^{\prime}=-x-\frac{\psi_{0}(x)}{\varphi_{-0}(x ; \mu)} .
$$

These operators factorize the Hamiltonian operators $H_{+}=$ $H_{-}+2$ and

$$
H_{\mu}=H_{+}+2 W^{\prime}=H_{-}-2\left(\ln \left(I_{0}(x)+\mu\right)\right)^{\prime \prime},
$$

$A_{\mu}^{+} A_{\mu}^{-}=H_{+}, A_{\mu}^{-} A_{\mu}^{+}=H_{\mu}$, and intertwine them, $A_{\mu}^{-} H_{+}=$ $H_{\mu} A_{\mu}^{-}, A_{\mu}^{+} H_{\mu}=H_{+} A_{\mu}^{+}$. In (5.8), the argument of logarithm is Wronskian of the states $\psi_{0}(x)$ and $\chi_{0}(x ; \mu): \operatorname{Wr}\left(\psi_{0}(x)\right.$, $\left.\chi_{0}(x ; \mu)\right)=I_{0}(x)+\mu$. Considering the second order differential operators given by a composition of the first order Darboux generators,

$$
\mathbb{A}_{\mu}^{-}=A_{\mu}^{-} a^{-}, \quad \mathbb{A}_{\mu}^{+}=a^{+} A_{\mu}^{+},
$$

we find that they intertwine the Hamiltonian operators $H_{-}=L-1$ and $H_{\mu}$,

$$
\mathbb{A}_{\mu}^{-} H_{-}=H_{\mu} \mathbb{A}_{\mu}^{-}, \quad \mathbb{A}_{\mu}^{+} H_{\mu}=H_{-} \mathbb{A}_{\mu}^{+},
$$

and also satisfy relations $\mathbb{A}_{\mu}^{+} \mathbb{A}_{\mu}^{-}=\left(H_{-}\right)^{2}, \mathbb{A}_{\mu}^{-} \mathbb{A}_{\mu}^{+}=\left(H_{\mu}\right)^{2}$. By construction, $\operatorname{ker}\left(\mathbb{A}_{\mu}^{-}\right)=\operatorname{span}\left\{\psi_{0}(x), \chi_{0}(x ; \mu)\right\}$. The Darboux-deformed oscillator system described by the Hamiltonian operator $H_{\mu}$ is completely isospectral to the system $H_{-}$. Its eigenstates with eigenvalues $E=2 n$, $n=1,2 \ldots$, are obtained by mapping the eigenstates $\psi_{n}(x)$ of the harmonic oscillator by the operator $\mathbb{A}_{\mu}^{-}$: $\psi_{n}(x) \mapsto \psi_{n}(x ; \mu)=\mathbb{A}_{\mu}^{-} \psi_{n}(x), H_{\mu} \psi_{n}(x ; \mu)=2 n \psi_{n}(x ; \mu)$. The (not normalized) ground state of zero energy of the system $H_{\mu}$ is described by wave function $\psi_{0}(x ; \mu)=\frac{1}{\varphi_{-0}(x ; \mu)}$, where $\varphi_{-0}(x ; \mu)$ is a wave function (5.4). It is obtained by application of the operator $\mathbb{A}_{\mu}^{-}$to the nonphysical eigenstate (5.2) of $H_{-}=L-1$ of zero energy, $\mathbb{A}_{\mu}^{-} \widetilde{\psi_{0}(x)}=-\psi_{0}(x ; \mu)$.

Thus, we obtained the completely isospectral pair of the Hamiltonian operators $H_{-}$and $H_{\mu}$, from which we compose the extended system described by the matrix Hamiltonian operator

$$
\mathcal{H}_{\mu}=\left(\begin{array}{cc}
H_{\mu} & 0 \\
0 & H_{-}
\end{array}\right)
$$

Its completely isospectral subsystems $H_{-}$and $H_{\mu}$, as we have seen, are intertwined by the second order operators (5.9) according to (5.10). On the other hand, the first order operators $A_{\mu}^{-}$and $A_{\mu}^{+}$intertwine $H_{+}=H_{-}+2$ and $H_{\mu}$. Therefore, these first order operators intertwine the subsystems $H_{-}$and $H_{\mu}$ of the extended system (5.11) but with a relative shift in comparison with (5.10),

$$
A_{\mu}^{-} H_{-}=\left(H_{\mu}-2\right) A_{\mu}^{-}, \quad A_{\mu}^{+}\left(H_{\mu}-2\right)=H_{-} A_{\mu}^{+} .
$$

From this construction we have two Darboux schemes: the scheme based on the ground eigenstate and Jordan state of the harmonic oscillator which produces the second order intertwining operators $\mathbb{A}_{\mu}^{ \pm}$. We denote such a scheme $\left(\psi_{0}(x), \chi_{0}(x ; \mu)\right)$. Another scheme is based on the nonphysical eigenstate $\varphi_{-0}(x ; \mu)$ of $L$ of eigenvalue -1 , which we denote as $\left(\varphi_{-0}(x ; \mu)\right)$. We can construct one more Darboux scheme $\left(\psi_{0}(x), \psi_{1}(x), a^{+} \chi_{0}(x ; \mu)\right)$ based on the physical eigenstates $\psi_{0}(x)$ and $\psi_{1}(x)$ and the state $a^{+} \chi_{0}(x ; \mu)$. This scheme gives rise to the third order intertwining operators $\mathcal{A}_{\mu}^{-}=A_{\mu}^{-}\left(a^{-}\right)^{2}=\mathbb{A}_{\mu}^{-} a^{-}$and $\mathcal{A}_{\mu}^{+}=\left(\mathcal{A}_{\mu}^{-}\right)^{\dagger}$. These operators also intertwine $H_{-}$and $H_{\mu}$ but with opposite relative shift in comparison with (5.12): $\quad \mathcal{A}_{\mu}^{-} H_{-}=\left(H_{\mu}+2\right) \mathcal{A}_{\mu}^{-}, \quad \mathcal{A}_{\mu}^{+}\left(H_{\mu}+2\right)=H_{-} \mathcal{A}_{\mu}^{+}$. Using the intertwining operators of these three Darboux schemes, we construct three pairs of antidiagonal (odd with respect to $\Gamma=\sigma_{3}$ ) operators

$$
\begin{gathered}
\mathcal{Q}_{\mu 1}=\left(\begin{array}{cc}
0 & \mathbb{A}_{\mu}^{-} \\
A_{\mu}^{+} & 0
\end{array}\right), \quad \mathcal{Q}_{\mu 2}=i \sigma_{3} \mathcal{Q}_{\mu 1}, \\
\mathcal{S}_{\mu 1}=\left(\begin{array}{cc}
0 & A_{\mu}^{-} \\
A_{\mu}^{+} & 0
\end{array}\right), \quad \mathcal{S}_{\mu 2}=i \sigma_{3} \mathcal{S}_{\mu 1}, \\
\mathcal{L}_{\mu 1}=\left(\begin{array}{cc}
0 & \mathcal{A}_{\mu}^{-} \\
\mathcal{A}_{\mu}^{+} & 0
\end{array}\right), \quad \mathcal{L}_{\mu 2}=i \sigma_{3} \mathcal{L}_{\mu 1} .
\end{gathered}
$$

Using the relations between the intertwining operators $\mathbb{A}_{\mu}^{-} A_{\mu}^{+}=A_{\mu}^{-} a^{-} A_{\mu}^{+}, \quad A_{\mu}^{+} \mathbb{A}_{\mu}^{-}=\left(H_{-}+2\right) a^{-}, \quad \mathcal{A}_{\mu}^{-} A_{\mu}^{+}=$ $A_{\mu}^{-}\left(a^{-}\right) A_{\mu}^{+}, A_{\mu}^{+} \mathcal{A}_{\mu}^{-}=\left(H_{-}+2\right)\left(a^{-}\right)^{2}$, we also construct diagonal (even) operators

$$
\begin{aligned}
\mathcal{C}_{\mu-} & =\left(\begin{array}{cc}
A^{-} a^{-} A^{+} & 0 \\
0 & \left(H_{-}+2\right) a^{-}
\end{array}\right), \\
\mathcal{J}_{\mu-} & =\left(\begin{array}{cc}
A^{-}\left(a^{-}\right)^{2} A^{+} & 0 \\
0 & \left(H_{-}+2\right)\left(a^{-}\right)^{2}
\end{array}\right),
\end{aligned}
$$

and Hermitian conjugate operators $\mathcal{C}_{\mu+}$ and $\mathcal{J}_{\mu+}$. With respect to the Hamiltonian $\mathcal{H}_{\mu}$, the only pair of timeindependent integrals are the supercharges $\mathcal{Q}_{\mu a}, a=1,2$. Other operators have to be dressed with the unitary evolution operator $U(t)=\exp \left(i \mathcal{H}_{\mu} t\right): \mathcal{S}_{\mu a} \mapsto U^{-1}(t) \mathcal{S}_{\mu a} U(t)$, etc., that gives us the corresponding dynamical integrals of motion. The obtained in such a way time-independent and dynamical integrals together with Hamiltonian operator $\mathcal{H}_{\mu}$ generate a kind of nonlinear superalgebra corresponding to a nonlinear deformation of the super-Schrödinger symmetry.

We are not interested here in explicit form of such a nonlinear superalgebra, but just note that when $\mu \rightarrow \pm \infty$, we have $(\ln (I(x)+\mu))^{\prime} \rightarrow 0$. As a result, in any of the two limits the Hamiltonian $H_{\mu}$ transforms into $H_{-}$, and the matrix Hamiltonian transforms into extended Hamiltonian 


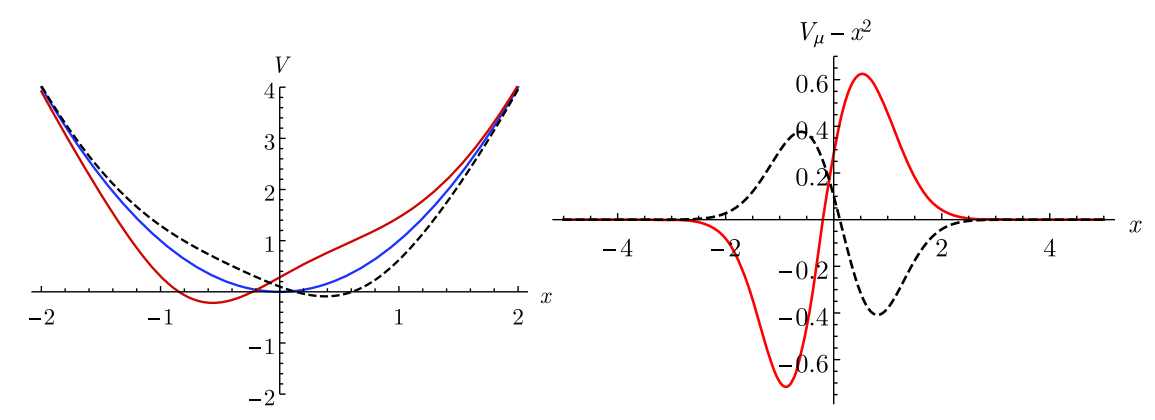

FIG. 1. On the left: Isospectrally deformed potential $V_{\mu}$ at $\mu=1$ and $\mu=-3$ is shown by continuous red and dashed black lines, respectively. On the right: The difference $V_{\mu}(x)-x^{2}$ given by the last term in Eq. (5.8) is shown for the same values $\mu=1$ and $\mu=-3$. With increasing value of modulus of the deformation parameter $\mu$ the amplitudes of minimum and maximum of the difference $V_{\mu}(x)-x^{2}$ decrease, and in both limits $\mu \rightarrow \pm \infty$ the deformed potential $V_{\mu}(x)$ transforms into harmonic potential $V=x^{2}$ shown on the left by continuous blue line.

(3.1) shifted for the minus unit matrix: $\mathcal{H}_{\mu} \rightarrow \mathcal{H}-\llbracket$. In this limit we also have $A_{\mu}^{ \pm} \rightarrow-a^{\mp}$, and find that the constructed operators transform as follows:

$$
\begin{aligned}
& \mathcal{Q}_{\mu 1} \rightarrow-(\mathcal{H}-1) \sigma_{1}, \quad \mathcal{Q}_{\mu 2} \rightarrow(\mathcal{H}-1) \sigma_{2}, \\
& \mathcal{S}_{\mu a} \rightarrow-\breve{\mathcal{S}}_{a}, \quad \mathcal{L}_{\mu a} \rightarrow-\left(\mathcal{H}-2+\sigma_{3}\right) \hat{\mathcal{Q}}_{a}, \\
& \mathcal{C}_{\mu-} \rightarrow\left(\mathcal{H}-\sigma_{3}\right) \mathcal{C}_{-}, \quad \mathcal{C}_{\mu+} \rightarrow \mathcal{C}_{+}\left(\mathcal{H}-\sigma_{3}\right), \\
& \mathcal{J}_{\mu-} \rightarrow\left(\mathcal{H}-\sigma_{3}\right) \mathcal{J}_{-}, \quad \mathcal{J}_{\mu+} \rightarrow \mathcal{J}_{+}\left(\mathcal{H}-\sigma_{3}\right) .
\end{aligned}
$$

In such a way we reproduce all the corresponding integrals of the system (3.1) that generate the super-extended Schrödinger symmetry lying behind the hidden superconformal symmetries $\mathfrak{o} \mathfrak{G} \mathfrak{p}(1 \mid 2)$ and $\mathfrak{o} \mathfrak{G} \mathfrak{p}(2 \mid 2)$ of a single quantum harmonic oscillator.

The isospectral deformation $V_{\mu}(x)$ of the harmonic oscillator potential is illustrated by Figure 1, while Figure 2 illustrates the action of the intertwining operators $\mathbb{A}_{\mu}^{ \pm}$and $\mathcal{A}_{\mu}^{ \pm}$.

In conclusion of this section we note that the Hamiltonian (5.11) and the second order intertwining operators $\mathbb{A}_{\mu}^{ \pm}$can be presented in alternative form which corresponds to the anomaly-free scheme of quantization of classical systems with second-order supersymmetry [15]. For this we introduce the quasiamplitude [16]

$$
\Xi(x)=\sqrt{\psi_{-0}(x) \varphi_{-0}(x ; \mu)} .
$$

It is a square root of the product of two nonphysical eigenstates of eigenvalue -1 of the quantum harmonic oscillator $L$. The rescaled function $\Xi(x) / \sqrt{\mu}$ transforms in the limit $\mu \rightarrow \pm \infty$ into the non-physical eigenstate $\psi_{-0}$. This function satisfies Ermakov-Pinney equation

$$
-\Xi^{\prime \prime}+\left(x^{2}+1\right) \Xi=\frac{1}{4 \Xi^{3}} .
$$

In terms of quasiamplitude, the first order differential operators

$$
A_{\Xi}^{-}=\Xi(x) \frac{d}{d x} \frac{1}{\Xi(x)}=\frac{d}{d x}-x-\mathcal{W}(x), \quad A_{\Xi}^{+}=\left(A_{\Xi}\right)^{\dagger}
$$

can be defined, where

$$
\mathcal{W}(x)=\frac{1}{2 \Xi^{2}(x)}=\frac{1}{2}\left(\ln \left(I_{0}(x)+\mu\right)\right)^{\prime} .
$$

Then the Hamiltonian $H_{\mu}$ and the intertwining operator $\mathbb{A}_{\mu}^{-}$ can be presented in the form

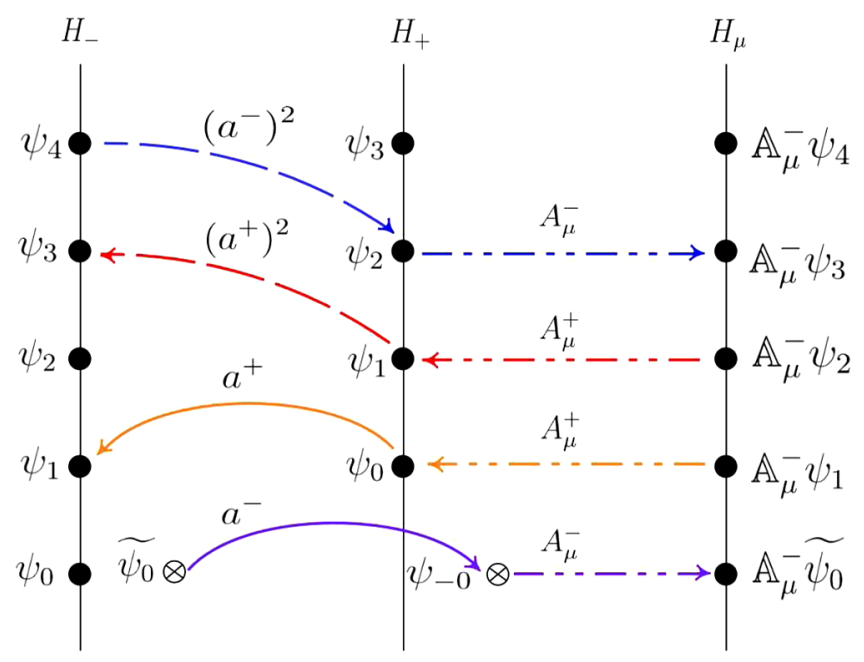

FIG. 2. Mapping of eigenstates of the systems $H_{-}$and $H_{\mu}$ by intertwining operators $\mathbb{A}_{\mu}^{ \pm}$and $\mathcal{A}_{\mu}^{ \pm}$via eigenstates of intermediate system $H_{+}$. The ground state $\mathbb{A}_{\mu}^{-} \widetilde{\psi_{0}}$ of $H_{\mu}$ is obtained by applying $\mathbb{A}_{\mu}^{-}$to nonphysical eigenstate $\psi_{0}$ of $H_{-}$. It also can be generated by a not shown here action of $\mathcal{A}_{\mu}^{-}$on nonphysical eigenstate $\widetilde{\psi_{1}}$ of $H_{-}$via nonphysical eigenstate $\psi_{-0}$ of $H_{+}$. 


$$
\begin{aligned}
& \mathcal{H}_{\mu}=A_{\Xi}^{-} A_{\Xi}^{+}+\mathcal{W}^{2}-2 \mathcal{W}^{\prime} \sigma_{3}, \\
& \mathbb{A}_{\mu}^{-}=-\left(A_{\Xi}^{-}-\mathcal{W}\right)\left(A_{\Xi}^{+}+\mathcal{W}\right) .
\end{aligned}
$$

Function $\mathcal{W}(x)$ in the anomaly-free scheme of quantization plays a role of superpotential for corresponding classical system with second order supersymmetry $[15,17,18]$.

\section{FREE PARTICLE LIMIT}

It is interesting to apply a limit of zero frequency to the constructions which have allowed us to clarify the nature of the hidden superconformal symmetry of the single quantum harmonic oscillator. In this way we identify a hidden superconformal symmetry of the free particle. A nontrivial point in this procedure is that it is necessary to make the appropriate different rescalings for different generators of super-Schrödinger symmetry of the extended quantum harmonic oscillator systems in order to obtain correctly their analogs for the corresponding (doubled) free particle system.

To start, let us restore the frequency parameter $\omega$ in the Hamiltonian of harmonic oscillator $L$ and in its ladder operators $a^{ \pm}$but maintaining the Planck constant $\hbar=1$ and mass parameter $m=\frac{1}{2}$ as before. We have

$$
\begin{aligned}
L \rightarrow L_{\omega} & =\omega\left(-\frac{1}{\omega} \frac{d^{2}}{d x^{2}}+\omega x^{2}\right)=\frac{1}{2} \omega\left\{a_{\omega}^{+}, a_{\omega}^{-}\right\}, \\
a^{ \pm} \rightarrow a_{\omega}^{ \pm} & =\mp \frac{1}{\sqrt{\omega}} \frac{d}{d x}+\sqrt{\omega} x,
\end{aligned}
$$

and $\left[L_{\omega}, a_{\omega}^{ \pm}\right]= \pm 2 \omega a_{\omega}^{ \pm}, \quad\left[L_{\omega},\left(a_{\omega}^{ \pm}\right)^{2}\right]= \pm 2 \omega\left(a_{\omega}^{ \pm}\right)^{2}$. We consider the application of the zero frequency limit for the extended system (3.1) and then make a comment for the case of the system (4.3). Applying the zero frequency limit to the matrix Hamiltonian operator (3.1) with restored according to (6.1) frequency, $\mathcal{H}_{\omega}$, it is convenient to rescale it, and we obtain

$$
\mathcal{H}_{0}=\left.2 \mathcal{H}_{\omega}\right|_{\omega \rightarrow 0}=\left(\begin{array}{cc}
H_{0} & 0 \\
0 & H_{0}
\end{array}\right), \quad H_{0}=-\frac{1}{2} \frac{d^{2}}{d x^{2}} .
$$

The nontrivial bosonic integrals for the system (6.2) are given by

$$
\begin{aligned}
& \mathcal{P}=\left.i \sqrt{2 \omega}\left(\mathcal{C}_{+}-\mathcal{C}_{-}\right)\right|_{\omega \rightarrow 0}=\frac{1}{\sqrt{2}}\left(\begin{array}{ll}
p & 0 \\
0 & p
\end{array}\right), \quad p=-i \frac{d}{d x}, \\
& \mathcal{G}=\left.\sqrt{\frac{2}{\omega}}\left(\mathcal{C}_{+}+\mathcal{C}_{-}\right)\right|_{\omega \rightarrow 0}=\frac{1}{\sqrt{2}}\left(\begin{array}{cc}
x-2 t p & 0 \\
0 & x-2 t p
\end{array}\right),
\end{aligned}
$$

$$
\begin{aligned}
\mathcal{D} & =\left.\frac{i}{2}\left(\mathcal{J}_{+}-\mathcal{J}_{-}\right)\right|_{\omega \rightarrow 0} \\
& =\frac{1}{4}\left(\begin{array}{cc}
\{x, p\}-4 t H_{0} & 0 \\
0 & \{x, p\}-4 t H_{0}
\end{array}\right), \\
\mathcal{K}=\left.\frac{1}{2 \omega}\left[\mathcal{J}_{+}+\mathcal{J}_{-}+\frac{2}{\omega} \mathcal{H}_{\omega}\right]\right|_{\omega \rightarrow 0} & \\
= & \frac{1}{2}\left(\begin{array}{cc}
x^{2}-2 t\{x, p\}+4 t^{2} H_{0} & 0 \\
0 & x^{2}-2 t\{x, p\}+4 t^{2} H_{0}
\end{array}\right) .
\end{aligned}
$$

These are generators of spatial translations $(\mathcal{P})$, of the Galilean boosts $(\mathcal{G})$, of dilatations $(\mathcal{D})$, and special conformal transformations $(\mathcal{K})$ of the doubled free particle system (6.2). The linear combinations $\mathcal{Q}_{+}-\mathcal{Q}_{-}$and $\mathcal{S}_{+}-$ $\mathcal{S}_{\text {- }}$ of the dynamical odd integrals of the system (3.1) produce time-independent supercharges for the system (6.2),

$$
\begin{gathered}
\pi_{1}=\left.i \sqrt{2 \omega}\left(\mathcal{Q}_{+}-\mathcal{Q}_{-}\right)\right|_{\omega \rightarrow 0}=\frac{1}{\sqrt{2}}\left(\begin{array}{ll}
0 & p \\
p & 0
\end{array}\right), \\
\pi_{2}=i \sigma_{3} \pi_{1}=\left.i \sqrt{2 \omega}\left(\mathcal{S}_{+}-\mathcal{S}_{-}\right)\right|_{\omega \rightarrow 0}=\frac{1}{\sqrt{2}}\left(\begin{array}{cc}
0 & i p \\
-i p & 0
\end{array}\right),
\end{gathered}
$$

while the linear combinations $\mathcal{Q}_{+}+\mathcal{Q}_{-}$and $\mathcal{S}_{+}+\mathcal{S}_{-}$give us the dynamical odd integrals,

$$
\begin{aligned}
& \xi_{1}=\left.\sqrt{\frac{2}{\omega}}\left(\mathcal{Q}_{+}+\mathcal{Q}_{-}\right)\right|_{\omega \rightarrow 0}=\frac{1}{\sqrt{2}}\left(\begin{array}{cc}
0 & x-2 t p \\
x-2 t p & 0
\end{array}\right) \\
& \xi_{2}=i \sigma_{3} \xi_{1}=\left.\sqrt{\frac{2}{\omega}}\left(\mathcal{S}_{+}+\mathcal{S}_{-}\right)\right|_{\omega \rightarrow 0} \\
&=\frac{i}{\sqrt{2}}\left(\begin{array}{cc}
0 & x-2 t p \\
-x+2 t p & 0
\end{array}\right) .
\end{aligned}
$$

Notice the difference in frequency factors before the corresponding linear combinations in (6.7), (6.8) and (6.9), (6.10). The time-independent even integrals $\mathcal{I}$ and $\mathcal{Z}$ and odd integrals $\Sigma_{a}, a=1,2$, of the system (3.1) are also the integrals for the system (6.2). All these operators generate the $\mathcal{N}=2$ super-Schrödinger algebra for the doubled free particle system (6.2) with the following nontrivial (anti)commutation relations:

$$
\begin{array}{ll}
{\left[\mathcal{D}, \mathcal{H}_{0}\right]=i \mathcal{H}_{0},} & {[\mathcal{D}, \mathcal{K}]=-i \mathcal{K},} \\
{\left[\mathcal{K}, \mathcal{H}_{0}\right]=2 i \mathcal{D},} & {[\mathcal{G}, \mathcal{P}]=2 i \mathcal{I},}
\end{array}
$$




$$
\begin{gathered}
{\left[\mathcal{H}_{0}, \mathcal{G}\right]=-i \mathcal{P}, \quad[\mathcal{K}, \mathcal{P}]=i \mathcal{G}} \\
{[\mathcal{D}, \mathcal{P}]=\frac{i}{2} \mathcal{P}, \quad[\mathcal{D}, \mathcal{G}]=-\frac{i}{2} \mathcal{G}} \\
{\left[\mathcal{D}, \pi_{a}\right]=\frac{i}{2} \pi_{a}, \quad\left[\mathcal{D}, \xi_{a}\right]=-\frac{i}{2} \xi_{a},} \\
{\left[\mathcal{Z}, \pi_{a}\right]=-\frac{i}{2} \epsilon_{a b} \pi_{b}, \quad\left[\mathcal{Z}, \xi_{a}\right]=-\frac{i}{2} \epsilon_{a b} \xi_{b},} \\
{\left[\mathcal{H}_{0}, \xi_{a}\right]=-i \pi_{a}, \quad\left[6, \pi_{a}\right]=i \xi_{a},} \\
{\left[\mathcal{Z}, \Sigma_{a}\right]=\frac{i}{2} \epsilon_{a b} \Sigma_{b}, \quad\left[\mathcal{P}, \pi_{a}\right]=-i \Sigma_{a},} \\
\left\{\Sigma_{a}, \pi_{b}\right\}=\delta_{a b} \mathcal{P}, \quad\left\{\Sigma_{a}, \xi_{a}\right]=i \Sigma_{a}, \\
\left\{\Sigma_{a}, \Sigma_{b}\right\}=2 \delta_{a b} \mathcal{I}, \quad \\
\left\{\pi_{a}, \pi_{b}\right\}=2 \delta_{a b} \mathcal{H} \mathcal{H}_{0}, \quad\left\{\xi_{a}, \xi_{b}\right\}=2 \delta_{a b} \mathcal{K}, \\
\left\{\pi_{a}, \xi_{b}\right\}=2 \delta_{a b} \mathcal{D}+2 \epsilon_{a b} \mathcal{Z} .
\end{gathered}
$$

Note that the superalgebra of the extended (doubled) quantum harmonic system (3.1) can be presented in the form (6.11)-(6.17) with the following correspondence between the generators:

$$
\begin{array}{rlrl}
\mathcal{H}_{0} & \leftrightarrow \mathcal{J}_{0}-\frac{1}{2}\left(\mathcal{J}_{+}+\mathcal{J}_{-}\right), \quad & \mathcal{K} \leftrightarrow \mathcal{J}_{0}+\frac{1}{2}\left(\mathcal{J}_{+}+\mathcal{J}_{-}\right), \\
\mathcal{D} & \leftrightarrow \frac{i}{2}\left(\mathcal{J}_{+}-\mathcal{J}_{-}\right) \\
\mathcal{P} & \leftrightarrow \sqrt{2} i\left(\mathcal{C}_{+}-\mathcal{C}_{-}\right), \quad \mathcal{G} \leftrightarrow \sqrt{2}\left(\mathcal{C}_{+}+\mathcal{C}_{-}\right), \\
\pi_{1} & \leftrightarrow \sqrt{2} i\left(\mathcal{Q}_{+}-\mathcal{Q}_{-}\right), & \pi_{2} \leftrightarrow \sqrt{2} i\left(\mathcal{S}_{+}-\mathcal{S}_{-}\right), & (6.20) \\
\xi_{1} & \leftrightarrow \sqrt{2}\left(\mathcal{Q}_{+}+\mathcal{Q}_{-}\right), & \xi_{2} \leftrightarrow \sqrt{2}\left(\mathcal{S}_{+}+\mathcal{S}_{-}\right),
\end{array}
$$

and with the same generators $\mathcal{Z}, \mathcal{I}$ and $\Sigma_{a}$ for both systems.

In the case of the supersymmetric system (4.3) after reconstruction of the frequency parameter we have

$$
\hat{\mathcal{H}}_{\omega}=\frac{1}{4}\left(\begin{array}{cc}
L_{\omega}+\omega & 0 \\
0 & L_{\omega}-\omega
\end{array}\right) .
$$

The zero frequency limit applied to this system produces the same doubled free particle system (6.2). This can also be understood by noting that after reconstruction of frequency, the superpotential in (4.3) takes a form $W_{\omega}=$ $\omega x$. In the zero frequency limit $W_{\omega} \rightarrow 0$ and $\hat{\mathcal{H}}_{\omega}=$ $-\frac{d^{2}}{d x^{2}}+W_{\omega}^{2}+W_{\omega}^{\prime} \sigma_{3} \rightarrow-\frac{d^{2}}{d x^{2}}$.

The application of the unitary nonlocal operator (3.15) to the odd generators $\pi_{a}$ and $\xi_{a}$ transforms them into operators of the diagonal form, and, particularly, $\pi_{1}$ and $\xi_{1}$ take the form of the operators $\mathcal{P}$ and $\mathcal{G}$. The transformed $\mathcal{P}$ and $\mathcal{G}$ take the anti-diagonal form, $\tilde{\mathcal{P}}=\pi_{1}, \tilde{\mathcal{G}}=\xi_{1}$. After projection to the proper subspace of eigenvalue +1 of $\sigma_{3}$, $\widetilde{\mathcal{X}_{i}} \mapsto \Pi_{+} \widetilde{\mathcal{X}_{i}} \Pi_{+}$, we left with a single free particle system $H_{0}$, whose hidden superconformal $\mathfrak{o} \mathfrak{g} \mathfrak{p}(1 \mid 2)$ symmetry is generated by local even integrals $H_{0}, D=\frac{1}{4}(\{x, p\}-$ $\left.4 t H_{0}\right), K=\frac{1}{2} x^{2}-t\{x, p\}+2 t^{2} H_{0}$, and by odd integrals $P=p, G=x-p t$, with reflection operator $\mathcal{R}$ playing the role of the $\mathbb{Z}_{2}$-grading operator. The inclusion of nonlocal even integral $Z=-\frac{1}{4} \mathcal{R}$ and nonlocal odd integrals $i \mathcal{R} P$, $i \mathcal{R} G$ extends the hidden superconformal $\mathfrak{o} \mathfrak{g} \mathfrak{p}(1 \mid 2)$ symmetry for the hidden superconformal $\mathfrak{o} \mathfrak{s} \mathfrak{p}(2 \mid 2)$ symmetry of the free particle. Explicit form the (anti)commutation relations can easily be identified from the corresponding relations from (6.11)-(6.17).

\section{DISCUSSION AND OUTLOOK}

The extended doubled quantum harmonic oscillator system (3.1) is not described by a usual construction of supersymmetric quantum mechanics. Nevertheless it is possible to obtain it in the following way by starting from the level of classical mechanics. Consider a classical system described by a Hamiltonian

$$
H=p^{2}+W^{2}+W^{\prime}\left[\theta^{+}, \theta^{-}\right]
$$

with superpotential $W(x)=\sqrt{x^{2}+c^{2}}$, where $c>0$ is a constant and $\theta^{+}$and $\theta^{-}=\left(\theta^{+}\right)^{*}$ are Grassmann variables being classical analogs of the fermion creation-annihilation operators with a nonzero Poisson bracket $\left\{\theta^{+}, \theta^{-}\right\}_{P B}=-i$. After quantization the odd variables $\theta^{ \pm}$transform into fermionic creation-annihilation operators which can be realized in terms of $\sigma$-matrices: $\theta^{ \pm} \rightarrow \sigma_{ \pm}=\frac{1}{2}\left(\sigma_{1} \pm i \sigma_{2}\right)$. A direct quantum analog of this system is a supersymmetric completely isospecral pair in the phase of spontaneously broken supersymmetry, with nonsingular superpartner potentials $V_{ \pm}=x^{2}+c^{2} \pm x / \sqrt{x^{2}+c^{2}}$. The spectrum of subsystems is different from that of the quantum harmonic oscillator. On the other hand, if before the quantization we realize a canonical transformation $x \rightarrow X=x+$ $N \partial G(x, p) / \partial p, p \rightarrow P=p-N \partial G(x, p) / \partial x, \theta^{ \pm} \rightarrow \Theta^{ \pm}=$ $e^{ \pm i G(x, p)} \theta^{ \pm}$, where $N=\theta^{+} \theta^{-}$and $G=\frac{1}{2} \arcsin \left(\left(p^{2}-\right.\right.$ $\left.\left.x^{2}-c^{2}\right) /\left(p^{2}+x^{2}+c^{2}\right)\right)$, we obtain the canonically equivalent form of the Hamiltonian $H=P^{2}+X^{2}+c^{2}$. In the canonically transformed system, the new classical Grassmann variables $\Theta^{ \pm}$completely decouple and are the odd integrals of motion with Poisson bracket $\left\{\Theta^{+}, \Theta^{-}\right\}_{P B}=-i$. The quantization of the canonically transformed system gives us exactly the extended quantum system (3.1) shifted just for the additive constant $c^{2}$.

The classical system given by the Hamiltonian $H=$ $p^{2}+W^{2}+2 W^{\prime}\left[\theta^{+}, \theta^{-}\right]$with a simply changed in comparison with (7.1) boson-fermion coupling constant in the last term is characterized at the classical level by the 
second-order supersymmetry $[17,18]$. The application of the anomaly-free scheme of quantization [15] to such a system with superpotential (5.23) produces the extended quantum system (5.11).

In Sec. IV, we generated superconformal symmetry via dual Darboux transformations. The analogous construction can be applied for investigation of superconformal symmetry in supersymmetric rationally extended quantum harmonic and isotonic oscillator systems [13]. The results of such investigation will be presented by us elsewhere.

We derived the hidden superconformal symmetry of the one-dimensional quantum harmonic oscillator and clarified its nature. It would be interesting to analyze from the same perspective the case of $d$-dimensional quantum harmonic oscillator systems which also are characterized by the corresponding generalizations of the hidden superconformal symmetry considered here $[6,7,9,19,20]$.
It is interesting to note that our construction of the one-parametric completely isospectral deformation of the quantum harmonic oscillator from Sec. V can be generalized by taking instead of the pair $\left(\psi_{0}, \chi_{0}(\mu)\right)$ of the seed states in the Darboux-Crum transformation the pair $\left(\psi_{n}\right.$, $\chi_{n}(\mu)$ ), where $\chi_{n}(\mu)=\chi_{n}(x ; \mu)$ is the Jordan state corresponding to eigenvalue $E_{n}$ [8]. In this case we shall obtain completely isospectral deformations of the harmonic oscillator potential of a more complicated form. The properties of the quantum systems obtained in such a way deserve a further investigation.

\section{ACKNOWLEDGMENTS}

L. I. acknowledges the CONICYT scholarship 21170053. M. S. P. acknowledges support from research project USA1555.
[1] E. Witten, Dynamical breaking of supersymmetry, Nucl. Phys. B188, 513 (1981); Constraints on supersymmetry breaking, Nucl. Phys. B202, 253 (1982).

[2] F. Cooper, A. Khare, and U. Sukhatme, Supersymmetry and quantum mechanics, Phys. Rep. 251, 267 (1995).

[3] M. S. Plyushchay, Supersymmetry without fermions, arXiv: hep-th/9404081; Deformed Heisenberg algebra, fractional spin fields and supersymmetry without fermions, Ann. Phys. (N.Y.) 245, 339 (1996).

[4] F. Correa and M. S. Plyushchay, Hidden supersymmetry in quantum bosonic systems, Ann. Phys. (Amsterdam) 322, 2493 (2007).

[5] F. Correa, V. Jakubsky, L. M. Nieto, and M. S. Plyushchay, Self-isospectrality, Special Supersymmetry, and their Effect on the Band Structure, Phys. Rev. Lett. 101, 030403 (2008).

[6] M. de Crombrugghe and V. Rittenberg, Supersymmetric quantum mechanics, Ann. Phys. (N.Y.) 151, 99 (1983).

[7] A. B. Balantekin, H. A. Schmitt, and B. R. Barrett, Coherent states for the harmonic oscillator representations of the orthosymplectic supergroup $\operatorname{Osp}(1 / 2 \mathrm{~N}, \mathrm{R})$, J. Math. Phys. (N.Y.) 29, 1634 (1988).

[8] J. F. Cariñena and M. S. Plyushchay, Ground-state isolation and discrete flows in a rationally extended quantum harmonic oscillator, Phys. Rev. D 94, 105022 (2016).

[9] R. Bonezzi, O. Corradini, E. Latini, and A. Waldron, Quantum mechanics and hidden superconformal symmetry, Phys. Rev. D 96, 126005 (2017).

[10] L. L. Foldy and S. A. Wouthuysen, On the Dirac theory of spin $1 / 2$ particle and its nonrelativistic limit, Phys. Rev. 78, 29 (1950).
[11] J. Beckers and V. Hussin, Dynamical supersymmetries of the harmonic oscillator, Phys. Lett. 118A, 319 (1986).

[12] J. Beckers, D. Dehin, and V, Hussin, Symmetries and supersymmetries of the quantum harmonic oscillator, J. Phys. A 20, 1137 (1987).

[13] J. F. Cariñena, L. Inzunza, and M. S. Plyushchay, Dualityinduced rational extensions of the isotonic oscillator, arXiv:1707.07357.

[14] A. Arancibia and M.S. Plyushchay, Transmutations of supersymmetry through soliton scattering, and selfconsistent condensates, Phys. Rev. D 90, 025008 (2014).

[15] M. S. Plyushchay, Schwarzian derivative treatment of the quantum second-order supersymmetry anomaly, and coupling-constant metamorphosis, Ann. Phys. (Amsterdam) 377, 164 (2017).

[16] Yu. V. Brezhnev, What does integrability of finite-gap or soliton potentials mean?, Phil. Trans. R. Soc. A 366, 923 (2008).

[17] M. Plyushchay, Hidden nonlinear supersymmetries in pure parabosonic systems, Int. J. Mod. Phys. A 15, 3679 (2000).

[18] S. M. Klishevich and M. S. Plyushchay, Nonlinear supersymmetry, quantum anomaly and quasiexactly solvable systems, Nucl. Phys. B606, 583 (2001).

[19] J. Beckers, D. Dehin, and V. Hussin, On the Heisenberg and orthosymplectic superalgebras of the harmonic oscillator, J. Math. Phys. (N.Y.) 29, 1705 (1988).

[20] A. B. Balantekin, O. Castaños, and M. Moshinsky, Relations between the nuclear shell model Hamiltonian and the orthosymplectic superalgebra Osp(1|2), Phys. Lett. B 284, 1 (1992). 